This manuscript is a preprint and has not been submitted for traditional publication yet, we have a few options in mind but have not formally submitted a manuscript. Please note that this article has not undergone peer-review and the manuscript has yet to be formally accepted for publication. Subsequent versions of this manuscript may have slightly different content. If accepted, the final version of this manuscript will be available via the 'Peer-reviewed Publication DOI' link on the right-hand side of this webpage. Please feel free to contact any of the authors; we welcome feedback. Example Citation: Jia D., The Aerodynamics of a Diabolo. 2021. 


\title{
The Aerodynamics of a Diabolo
}

\author{
Darren $\mathrm{Jia}^{1}$
}

September 19, 2021

\begin{abstract}
Diabolo is a popular game in which the object can be spun at up to speeds of $5000 \mathrm{rpm}$. This high spin velocity gives the diabolo the necessary angular momentum to remain stable. The shape of the diabolo generates an interesting air flow pattern. The viscous air applies a resistive torque on the fast spinning diabolo. Through computational fluid dynamics (CFD) simulations it's shown that the resistive torque has an interesting dependence on the angular speed of the diabolo. Further, the geometric shape of the diabolo affects the dependence of torque on angular speed.
\end{abstract}

1

\section{Introduction}

The aerodynamics around spinning objects has long been studied using CFD and experimental techniques due to its complexity and a lack of analytical solution for the turbulent nature of flows involved. Objects of particular interest include cylinders [1] and spheres [2] 3] 44. Improved understanding of the aerodynamics of spinning and moving spherical objects lends to better design of sports objects such as cricket balls [5, golf balls [6], [7], [8], and soccer balls [9], [10], [11, and [12]. For example, it's long been known that the dimpled surface of a golf ball [7] 8 will disrupt laminar flow around the golf ball and cause the onset of turbulent flow to happen near the leading edge of the golf ball. Such early

\footnotetext{
${ }^{1}$ darrenjia@bernardsboe.com, Ridge High School, NJ $U S A$
}

onset of turbulent air flow significantly reduces the air resistance so the golf ball can fly further.

A traditional East Asian past time involves spinning a diabolo on a thin thread while the person performs many challenging movements. The aerodynamics aspect of a spinning diabolo has not been paid much tension in the past. In this work, we present a set of preliminary results concerning the aerodynamics of a spinning diabolo and the relationship between the spin angular velocity of the diabolo and the torque applied on the diabolo due to air resistance.

\section{Simulation Setup}

The aerodynamics of a spinning diabolo is simulated using the open source CFD software OpenFOAM [13 14] 15. We have previously implemented a solver [16 based on finite difference methods which suffered from the problem that the grid resolution is insufficient to resolve the boundary layer behavior near the solid walls of 
the diabolo.

OpenFOAM is a popular open source CFD package that supports a finite volume [13] approach to aerodynamics simulation. In addition, the finite volume of the mesh [17] is adaptive and can be refined near the walls of the diabolo to capture the details of the flow field near the wall, where large gradient of the flow field variables is present.

To set up the simulation, we first create a geometry of the diabolo using blender (open source CAD software) [18]. Then a finite volume mesh with refinement zone near the diabolo is created to be used in the OpenFOAM solver.

\subsection{Geometry of a Diabolo}

We used the measurements of a Mister Babache [19] diabolo along with a reference image of a Flight Lander diabolo to create a diabolo geometry in blender as shown in figure 1 .

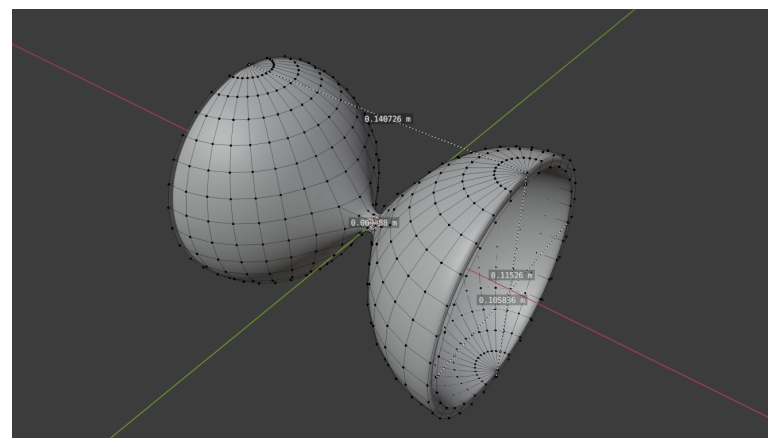

Figure 1: Geometry of the diabolo used in simulation: length $14.07 \mathrm{~cm}$, inner diameter $10.58 \mathrm{~cm}$, outer diameter: $11.53 \mathrm{~cm}$, narrow section diameter: $0.95 \mathrm{~cm}$

\subsection{Meshing}

As shown in figure 2, a refined boundary layer is formed around the wall of the diabolo. Then a refined mesh in the shape of a cylinder surrounds the diabolo. The radius of this refined mesh is about $10 \mathrm{~cm}$. The resolution of the mesh beyond the cylindrical refinement zone is lower.

On the sectional view of the mesh, the largest cells have a resolution of $6.45 * 10^{-3} \times 1.85 * 10^{-2}$ in meters. Each refinement level increases the resolution by a scale of 2 . Near the diabolo, the resolution becomes $3.23 * 10^{-3} \times 9.23 * 10^{-3}$ meter. Finally the boundary layer mesh has a highest resolution of $1.62 * 10^{-3} \times 4.61 * 10^{-3}$ meter. Overall the mesh used in the simulation for base diabolo geometry has 127313 cells.

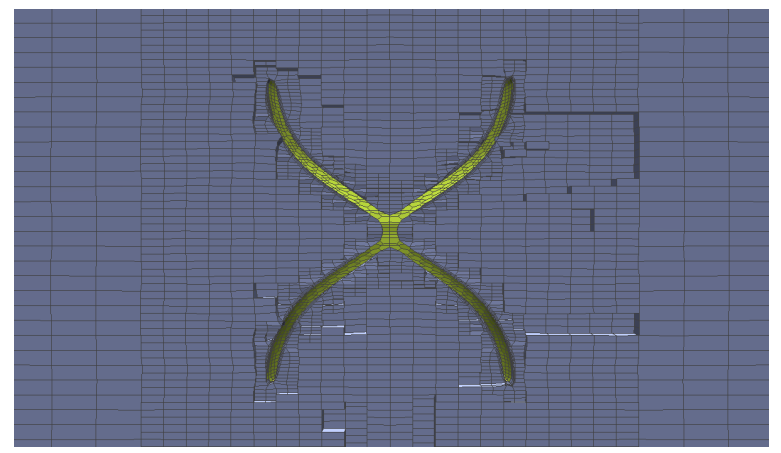

Figure 2: Meshing of the diabolo: the resolution of the mesh increases near the wall of the diabolo, including the refined boundary layer on the diabolo.

\subsection{Solver}

OpenFOAM SIMPLE solver uses a finite volume [13] approach to solve the steady-state incompressible viscous fluid flow [14] [15]. This is a suitable algorithm for the diabolo simulation as we are looking for a flow field solution when 
the angular speed of the diabolo becomes relatively steady. The governing equations of the incompressible viscous fluids are the typical incompressible steady Navier-Stokes equations:

$$
\begin{array}{r}
\nabla \vec{v}=0 \\
\nabla \cdot(\vec{v} \otimes \vec{v})=-\nabla p+\nabla(\nu \nabla \vec{v})
\end{array}
$$

In the SIMPLE algorithm, the pressurevelocity coupled equation is solved by decoupling the pressure and momentum fields through predictor-corrector steps. During the momentum predictor step, $H$ matrix is solved from the momentum equation which has been matrixized on the finite volume mesh.

$$
\begin{array}{r}
\nabla \cdot(\vec{v} \otimes \vec{v})-\nabla(\nu \nabla \vec{v})=-\nabla p \\
M V=-\nabla P \\
A=\operatorname{diag}(M) \\
H=A V-M V \\
A V-H=-\nabla P
\end{array}
$$

Now we can start the iterative process solving for pressure $P$ and velocity $V$. Start with the momentum equation,

$$
V=A^{-1} H-A^{-1} \nabla P
$$

Substituting the $V$ equation into continuity equation leads to a Poisson equation of pressure that can be solved by under relaxation method.

$$
\begin{array}{r}
\nabla V=\nabla\left(A^{-1} H-A^{-1} \nabla P\right)=0 \\
\nabla\left(A^{-1} \nabla P\right)=\nabla\left(A^{-1} H\right)
\end{array}
$$

In the predictor step, the momentum equation is solved using initial pressure $P$ and velocity boundary condition to find velocity $V$

$$
M V=-\nabla P
$$

The velocity field $V$ is then used in the Poisson equation to solve pressure $P$

$$
\nabla\left(A^{-1} \nabla P\right)=\nabla\left(A^{-1} H\right)
$$

The pressure field is then used in equation ( 8) to correct $V$ at the boundary. This is the corrector step. This process is iterated until a solution of $V$ and $P$ converges for the computational domain.

In this particular simulation, the $k-\epsilon$ [20] turbulence mode is enabled. The Reynolds number $R e=\frac{v L}{\nu}$ for a spinning diabolo at hundreds of $\mathrm{rad} / \mathrm{s}$ teogether with the geometry we used far exceeds the laminar regime of air flow.

\subsection{Flow pattern}

The contour plots in figure 3 show pressure, speed, and vorticity when the diabolo spins at $\omega=600 \mathrm{rad} / \mathrm{s}$. We clearly observe the presence of vortex shedding with its signature wavy pattern. As the angular velocity increases, the magnitude of pressure change from reference pressure, air speed, vorticity and wall shear stress all increase. The high pressure regions at the top and bottom of the computational domain are artifacts of the simulation where the mesh cells between the rotating refinement mesh and outer boundary of the computational domain rotate relative to the refinement geometry. This artifact can be more readily seen by examining the z-component of velocity which are opposite in sign above and below the diabolo. Further, the distance between origin (center of the diabolo) and the inner boundary of the high speed region above or below is exactly the radius of the cylindrical refinement mesh. The flow field beyond the refinement mesh is not analyzed due to the artificial nature of the result from OpenFOAM in this region. 

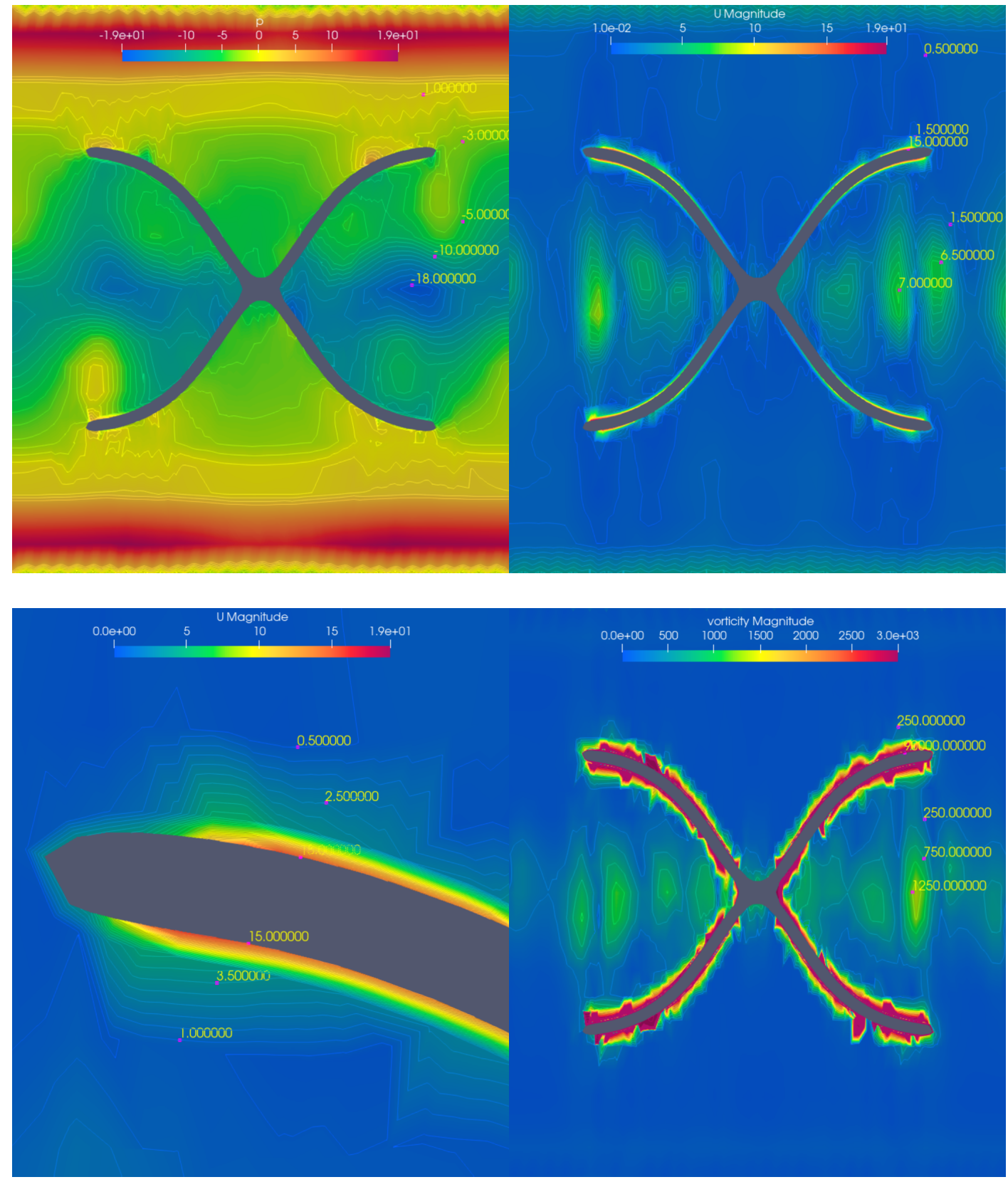

Figure 3: Air pressure (top left), speed (top right), close up speed near the wall of the diabolo (bottom left), and vorticity (bottom right) at $\omega=600 \mathrm{rad} / \mathrm{s}$. 


\subsection{Torque}

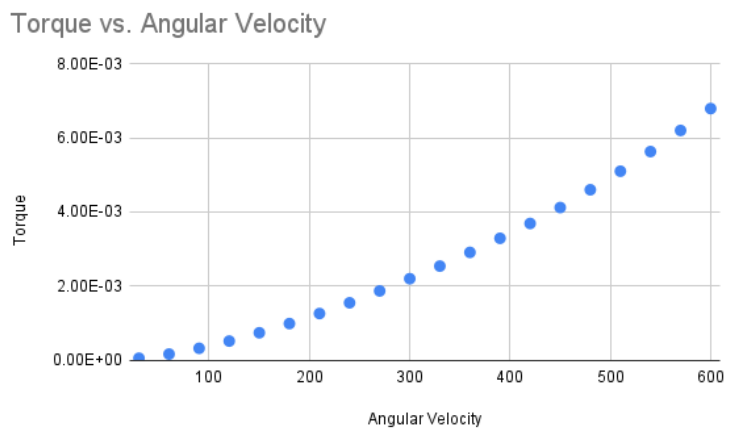

Figure 4: Torque on the base length diabolo model over varying angular velocities. As the angular velocity increases from 0 to $600 \mathrm{rad} / \mathrm{s}$, torque increases from 0 to $0.007 \mathrm{Nm}$.

\section{Ln Torque vs. Ln Omega}

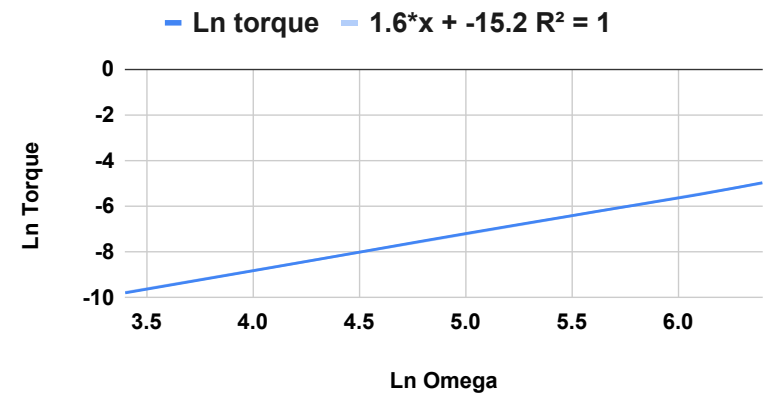

Figure 5: A log scale graph of the Torque vs $\omega$ data is shown here. Using the relationship found by the trendline, an equation can be created relating Angular Velocity and Torque. $\tau=$ $2.5 \cdot 10^{-7} \cdot \omega^{1.6}$.

As the diabolo is spinning, air friction applies a resistive torque on the diabolo due to wall shear stress. As time passes, the torque exerted on the diabolo stabilizes and converges on a value from the elliptic solver in OpenFOAM, allowing us to record the torque for any given angular velocity. We simulated the diabolo rotating along its lengthwise axis between $30 \mathrm{rad} / \mathrm{s}$ and $600 \mathrm{rad} / \mathrm{s}$ with an interval of $30 \mathrm{rad} / \mathrm{s}$ between data points. Figure 4 shows how the torque calculated along this axis varies as a function of angular velocity $\omega$ based on the measured values shown in table 1. The smooth pattern of the curve suggests a power law relationship. By applying logarithmic functions of the torque and angular velocity values, we can produce the relationship $\ln (\tau)=b \cdot \ln (\omega)+a$. This further leads to the following relationship

$$
\tau=\alpha \omega^{\beta}
$$

As shown figure 6, this equation correctly models the torque found through OpenFOAM simulation with high correlation. For the base diabolo geometry, the two coefficients through regression are $\alpha=2.5 \cdot 10^{-7}$ and $\beta=1.6 \mathrm{We}$ first use a base diablo geometry configuration to examine the torque-angular velocity relationship. In the next section, the geometry of the diabolo is varied and the effect of geometry on the torque-angular velocity is further investigated.

\section{Effect of geometry on aerody- namics}

After establishing the power law relationship between torque and angular velocity, we now modify the base geometry of the diabolo by scaling the diabolo model. Diabolos with varying lengths are created by scaling the diabolo along its lengthwise axis, while keeping the radius of the diabolo the same. The length scales are shown in table 2. We can then use these ge- 


\begin{tabular}{|c|c|}
\hline$\omega(\mathrm{rad} / \mathrm{s})$ & $\tau(\mathrm{N} \mathrm{m})$ \\
\hline 30 & $5.42 \mathrm{E}-05$ \\
\hline 60 & $1.66 \mathrm{E}-04$ \\
\hline 90 & $3.22 \mathrm{E}-04$ \\
\hline 120 & $5.17 \mathrm{E}-04$ \\
\hline 150 & $7.41 \mathrm{E}-04$ \\
\hline 180 & $9.89 \mathrm{E}-04$ \\
\hline 210 & $1.26 \mathrm{E}-03$ \\
\hline 240 & $1.55 \mathrm{E}-03$ \\
\hline 270 & $1.87 \mathrm{E}-03$ \\
\hline 300 & $2.20 \mathrm{E}-03$ \\
\hline 360 & $2.91 \mathrm{E}-03$ \\
\hline 390 & $3.29 \mathrm{E}-03$ \\
\hline 420 & $3.69 \mathrm{E}-03$ \\
\hline 450 & $4.12 \mathrm{E}-03$ \\
\hline 480 & $4.60 \mathrm{E}-03$ \\
\hline 510 & $5.10 \mathrm{E}-03$ \\
\hline 540 & $5.63 \mathrm{E}-03$ \\
\hline 570 & $6.20 \mathrm{E}-03$ \\
\hline 600 & $6.79 \mathrm{E}-03$ \\
\hline
\end{tabular}

Table 1: Torque calculated from wall shear stress in OpenFOAM as a function of the diabolo's spin angular velocity.

ometries to examine the effect of length on the torque-angular velocity relationship.

Similarly, diabolos of varying radii are created by scaling the diabolo radially, while keeping the length of the diabolo constant. The outer radius, inner radius, and narrow section of the diabolo are updated. The radius scales are shown in table 3. These geometries are used to examine the effect of radius on the torque-angular velocity relationship.

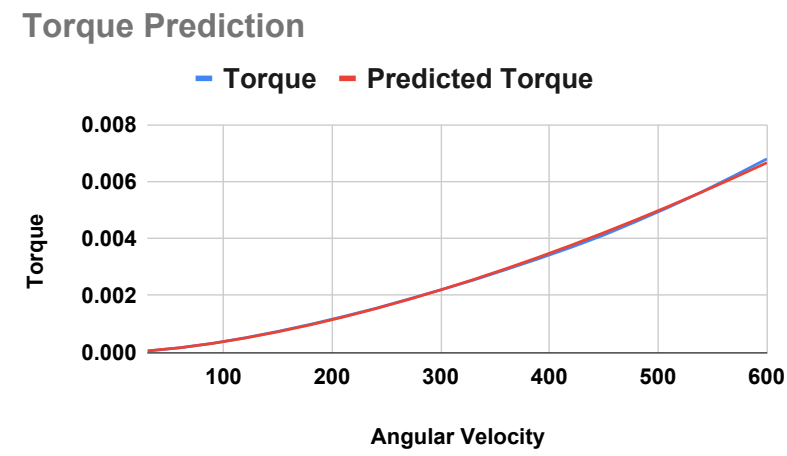

Figure 6: The simulated torque found in OpenFOAM and the predicted torque created by our equation match well.

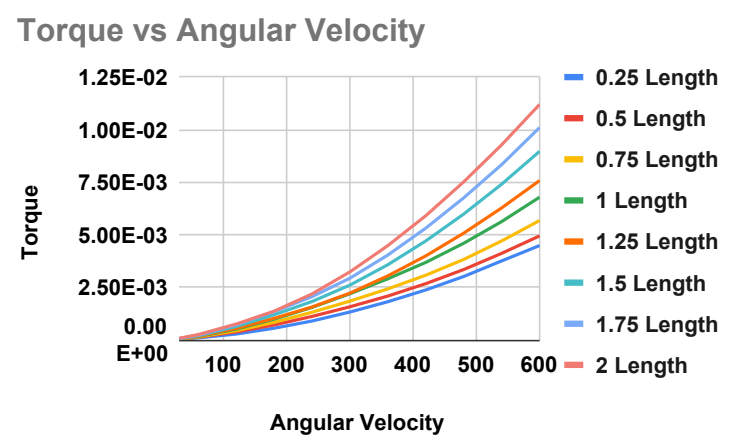

Figure 7: Torque as a function of angular velocity for varying diabolo lengths.

\section{$3.1 \quad$ Effect of length}

As shown in figure 7, increasing the length of the diabolo increases the torque exerted on it. The relation between torque and angular velocity remains the same $\left(\tau=\alpha \omega^{\beta}\right)$ however the values of $\alpha$ and $\beta$ vary with different diabolo lengths. The value of alpha increases linearly with diabolo length as shown in table 2, giving us the following relationship on $\alpha$ 


\begin{tabular}{|c|c|c|}
\hline Length Scale (dimensionless) & $\alpha(\mathrm{N} \mathrm{m})$ & $\beta$ (dimensionless) \\
\hline 0.25 & $1.38 \mathrm{E}-07$ & 1.613 \\
\hline 0.5 & $1.95 \mathrm{E}-07$ & 1.580 \\
\hline 0.75 & $2.11 \mathrm{E}-07$ & 1.592 \\
\hline 1 & $2.41 \mathrm{E}-07$ & 1.600 \\
\hline 1.25 & $2.47 \mathrm{E}-07$ & 1.606 \\
\hline 1.5 & $2.62 \mathrm{E}-07$ & 1.623 \\
\hline 1.75 & $2.80 \mathrm{E}-07$ & 1.633 \\
\hline 2 & $3.14 \mathrm{E}-07$ & 1.628 \\
\hline
\end{tabular}

Table 2: $\alpha$ and $\beta$ as a function of varying length scale from the base diabolo geometry. $\alpha$ increases due to increased contact surface area. $\beta$ stays around 1.6 for all lengths suggesting length has no effect on $\beta$. Length scale is the ratio between varied lengths against base diabolo length.

\begin{tabular}{|c|c|c|}
\hline Radius Scale (dimensionless) & $\alpha(\mathrm{N} \mathrm{m})$ & $\beta$ (dimensionless) \\
\hline 0.25 & $2.82 \mathrm{E}-09$ & 1.529 \\
\hline 0.5 & $2.17 \mathrm{E}-08$ & 1.584 \\
\hline 0.75 & $8.17 \mathrm{E}-08$ & 1.596 \\
\hline 1 & $2.41 \mathrm{E}-07$ & 1.599 \\
\hline 1.25 & $4.67 \mathrm{E}-07$ & 1.618 \\
\hline 1.5 & $8.04 \mathrm{E}-07$ & 1.658 \\
\hline 1.75 & $1.45 \mathrm{E}-06$ & 1.665 \\
\hline 2 & $2.21 \mathrm{E}-06$ & 1.703 \\
\hline
\end{tabular}

Table 3: $\alpha$ and $\beta$ as a function varying radius from the base diabolo geometry. Both variables increase with radius scale. Radius scale is the ratio between varied radii against base diabolo radius.

$$
\alpha=8.64 \cdot 10^{-8} \cdot L+1.39 \cdot 10^{-7}
$$

The value of $\beta$ stays around 1.6 as shown in table 2. There is no strong correlation between $\beta$ and length which suggests that $\beta$ remains constant at about 1.6 for all lengths.

Furthermore, a relationship between torque and length can be derived when $\omega$ is held constant. The torque exerted on the diabolo increases linearly with diabolo length when angular velocity is $600 \mathrm{rad} / \mathrm{s}$ with relation $\tau=$

$3.97 \cdot 10^{-3} \cdot L+3 \cdot 10^{-3}$.

A similar linear relationship for other angular velocities exists between length $L$ and torque $\tau$. The torque on the diabolo can be expressed more generally as

$$
\tau=a(\omega) \cdot L+b(\omega)
$$

where $a(\omega)$ and $b(\omega)$ are regressed against $\omega$ to produce the following equations

$$
a=9.21 \cdot 10^{-8} \cdot \omega^{1.66}
$$




$$
b=1.37 \cdot 10^{-7} \cdot \omega^{1.55}
$$

\subsection{Effect of radius}

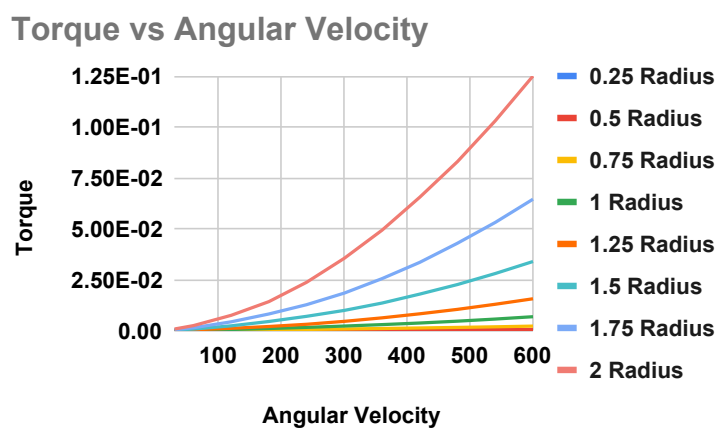

Figure 8: Torque as a function of angular velocity for varying radii.

As shown in figure 8 , increasing the radius of the diabolo increases the torque exerted as expected. The general relation between torque and angular velocity remains the same $\tau=\alpha \omega^{\beta}$.

The value for $\alpha$ increases as radius increases as shown in table 3 yielding the equation after regression

$$
\alpha=2.27 \cdot 10^{-7} \cdot r^{3.23}
$$

The value for $\beta$ increases linearly with radius as shown in table 3 , giving

$$
\beta=0.0869 r+1.52
$$

A similar relation can be derived between torque and radius when $\omega$ is held constant. The torque exerted on the diabolo when the angular velocity is held at $600 \mathrm{rad} / \mathrm{s}$ increases with radius, creating a smooth curve with relation $\tau=7.60 \cdot 10^{-3} \cdot r^{3.72}$
We see this same smooth curve for every other angular velocity. The torque dependence on radius $r$ can then be expressed more generally as

$$
\tau=a(\omega) \cdot r^{b(\omega)}
$$

where $a(\omega)$ is regressed against $\omega$ to produce

$$
a=3.38 \cdot 10^{-7} \cdot \omega^{1.56}
$$

$b(\omega)$ increases from 3.46 to 3.72 as $\omega$ increases from $30 \mathrm{rad} / \mathrm{s}$ to $600 \mathrm{rad} / \mathrm{s}$ with a correlation of 0.793 from linear regression.

\section{Conclusion and future work}

By varying the angular speed of the base diabolo geometry, we have observed a power law relationship between torque and angular velocity $\tau=\alpha \omega^{\beta}$. By further examining the dependence of torque on the shape of the diabolo, we can conclude that both coefficients are functions of the geometry of the diabolo.

The diabolo used in our simulation has a smooth wall which could perform better if we used a dimpled surface to reduce air resistance. We are not aware of any diabolos made of a dimpled surface. It'll be interesting to further investigate the effect of dimpled wall on the aerodynamics of diabolo.

One critical element missing from this work is experimental validation of the dependence found from CFD simulation. We suggest setting up experiments to verify if the predicted torqueangular velocity relationship agrees with observation. One approach is to measure the mechanical power required to sustain a constant angular speed described by equation 22 .

$$
P=\tau \omega=\alpha \omega^{\beta} \cdot \omega=\alpha \omega^{\beta+1}
$$


By measuring the power of motor needed to sustain the diabolo at varying angular velocity, we can then verify if the coefficients $\alpha$ and $\beta$ agrees with CFD simulation.

A second approach is to measure the angular deaccleration of the diabolo as a result of the viscous torque from air in the spinning diabolo. The measured deacceleration and the rotational inertia of the diabolo yields measured torque which then can be compared with predicted $\tau-\omega$ relationship.

$$
\begin{gathered}
\tau=I \frac{d \omega}{d t}=-\alpha \omega^{\beta} \\
\frac{d \omega}{d t}=-\frac{\alpha}{I} \omega^{\beta} \\
\omega(t)=\left((\beta-1) \frac{\alpha}{I} t\right)^{\frac{1}{1-\beta}}
\end{gathered}
$$

By measuring how $\omega$ of a spinning diabolo slows down and comparing with equation 23 , we can experimentally determine $\alpha$ and $\beta$ then compare with the simulated result from OpenFOAM.

It would be interesting to set up the above experiments using spinning diabolos to measure the dependence of torque on angular velocity and compare it with the result simulated from OpenFOAM. The comparison will help us better understand the strengths and weaknesses of OpenFOAM's result in this study.

\section{References}

[1] Kim HC, et al, "Three-dimensional flow analysis around a cylinder with dimples", Asia-Pacific congress on sports technology, 2005

[2] Archenbach E., "Experiments on the flow past spheres at very high Reynolds numbers", J Fluid Mech 54, 1972, 565-75

[3] Taneda S., "Visual observations on the flow past spheres at Reynods numbers between $10^{4}$ and $10^{6}$ ", J Fluid Mech., 85, 1978, 187-92
[4] Constantinescu GS, et al, "Turbulence modelling applied to flow over a sphere", AIAA J, 41(9), 2003, $1733-43$

[5] Penrose JMT, et al, "Cricket ball swing: a preliminary analysis using CFD" The engineering of sport, Sheffield, UK, 1996

[6] TIng LL., "Application of CFD technology analysing the 3D aerodynamic behavior of dimpled golf balls", ASME international mechanical engineering congress and exposition, 2002, New Orleans, Louisiana.

[7] Ting LL., "Effect of teardrop shaped dimple design on the golfball aerodynamic performance", ISEA 5th engineering of sport conference, 2004, International Sports Engineering Association.

[8] Aoki K, et al, "Effect of the dimple structure on the flying characteristics and flow patterns of a golf ball", 2004 "ISEA 5th engineering of sport conference. International Sports Engineering Association.

[9] Carre MJ, et al, "The curve kick of a football 2: flight through the air", Sports Eng., 5, 2002, 183-92

[10] Barber S., "The aerodynamics of association footballs", Mechanical engineering, University of Sheffield, 2007

[11] Barber S.,et al, "Sports ball aerodynamics: A numerical study of the erratic motion of soccer balls", Computers \& Fluids, 38, (2009) 1091-100

[12] Asai T., et al, "A fundamental study on aerodynamics of soccer ball", Proceedings of 83rd Japan society of mechanical engineering conference (Fluid engineering division), 2005

[13] Jasak H., "Error Analysis and Estimation for the Finite Volume Method with applications to Fluid Flows", Direct, M

[14] H. K. Versteeg and W. Malalasekera, An Introduction to Computational Fluid Dynamics: The Finite Volume Method Longman Scientific and Technical, 1995.

[15] J. H. Ferziger and M. Peric', Computational Methods for Fluid Dynamics Springer, Berlin, 1996. 
[16] H. Alan and F. Liu, Computational Fluid Dynamics simulation of a spinning diabolo, Private communication, 2020.

[17] Owen, S. J. 1998. A survey of unstructured mesh generation technology. In Proc. 7th Inter. Mesh. Roundtable, 239-267.

[18] Community, B. O. (2018). Blender - a 3D modelling and rendering package. Stichting Blender Foundation, Amsterdam. Retrieved from http://www.blender.org

[19] https://www.diabolotricks.com/wii-BabacheFin esse.html

[20] Henk Kaarle Versteeg, Weeratunge Malalasekera (2007). An Introduction to Computational Fluid Dynamics: The Finite Volume Method. 